NOTE: This is a pre-publication manuscript version of a published article. This paper is not the copy of record and may not exactly replicate the authoritative document published in the journal.

\title{
Modeling Cultural Change: Computational Models of Interpersonal Influence Dynamics Can Yield New Insights about How Cultures Change, Which Cultures Change More Rapidly Than Others, And Why
}

\author{
Mark Schaller \\ University of British Columbia
}

\author{
Michael Muthukrishna \\ London School of Economics and Political Science
}

In press, American Psychologist

\begin{abstract}
Cultural change can occur as an emergent consequence of social influence dynamics within cultural populations. These influence dynamics are complex, and formal modeling methods — such as agent-based models - are a useful means of predicting implications for cultural change. These models may be especially useful if they not only model the psychological outcomes of interpersonal influence, but also model social network structures within a culture. When combined, these components provide a flexible modeling framework that allows other variables to also be modeled for the purposes of predicting plausible implications for cultural change. The article illustrates this approach by summarizing recent research that employed these methods to model cross-cultural differences in the pace of cultural change. The article then identifies additional variables that could potentially be modeled within this conceptual framework, in order to produce additional insights — and additional new hypotheses — about different circumstances associated with different patterns of cultural change.

Keywords: culture, cultural change, social influence, social networks, computational modeling

Public Significance Statement: Cultural norms - in the form of widespread beliefs and popular behavioral choices - affect political elections, public policy decisions, and public health outcomes. But norms can change, with consequential implications. This article describes how computational models of social influence processes provide a means to better understand, and predict, consequential cultural changes.
\end{abstract}

Cultures are defined by many things - the values that people endorse, the technologies that people use, the foods that people eat, the music that they listen to, the countless specific beliefs and expectations and rituals and norms that govern the countless interactions that they have with other people. Over time these things change. People adopt new values, new technologies, new tastes, new beliefs and expectations, and cultures change accordinglysometimes slowly, and sometimes with surprising speed. These cultural changes are an important topic for psychological inquiry, not only because cultural changes affect the thoughts and actions of the people who comprise those cultures, but also because these changes are themselves emergent consequences of the thoughts and actions of those people.

Although cultural change is deeply psychological, the topic does not lend itself easily to psychological analysis. Most psychological theories and research methods are directed toward phenomena operating solely at the individual level of analysis, with simple unidirectional relations between causes and effects. But cultural change is a population-level phenomenon, characterized by highly dynamic bidirectional causal relations between the attributes of individuals and the cultural norms that they simultaneously respond to and create. It is no small 
2

challenge to describe cultural changes that have already occurred, or to identify events and circumstances that might plausibly have played some role in producing those changes (Muthukrishna, Henrich, Slingerland, 2021). It is perhaps even more challenging to explain - in psychological terms - why those changes occurred, or to predict changes that might plausibly occur in the future. In order to meet that challenge, an expanded repertoire of conceptual and methodological tools may be required.

With that goal in mind - and also mindful of the maxim "There is nothing so practical as a good theory" (commonly attributed to Kurt Lewin ${ }^{1}$ ) and its shrewd companion "There is nothing so theoretical as a good method" (Greenwald, 2012)—we suggest that research on cultural change can be abetted by a combination of rigorous theorizing and methodological innovation. This article describes one useful theoretical framework that can be applied to the study of cultural change. This framework focuses on social influence processes, and the culturallevel consequences that occur over time as people interact with, and exert influence on, each other. This article also describes a computational modeling methodology that allows this theoretical framework to be articulated in a rigorous and precise manner, in order to address questions about how cultures change, which cultures change more rapidly than others, and why.

\section{Models of Social Influence and Cultural Change}

Many different kinds of processes can contribute to cultural change (Varnum \& Grossman, 2017a). For example, one broad line of inquiry is based on the premise that cultural norms are adapted to local ecologies (Sng, Neuberg, Varnum, \& Kenrick, 2018); consequently, when specific ecological circumstances change, specific cultural changes may follow (Grossmann \& Varnum, 2015; Santos, Varnum, \& Grossmann, 2017; Varnum \& Grossmann, 2017b). That perspective is not the focus here (although we discuss ecological variables toward the end of this article). Our focus is instead on a complementary perspective that emphasizes the psychology of social influence.

Most psychological research on social influence focuses on brief snapshots of unidirectional influence involving small numbers of people. In reality, social influence is much more dynamic. Just as any single social interaction presents an opportunity for one person to influence another, it also offers a reciprocal opportunity for the latter to exert influence on the former. As individuals go about their lives, they have lots of interactions with lots of people; and so when influence does occur (e.g., when individuals actually experience changes in their attitudes or beliefs) those changes have the potential to be "contagious" - to spread through additional interactions with additional people (Berger, 2013). To the extent that these psychological changes do spread widely, cultures change; and to the extent that these psychological changes spread more rapidly, cultures change more rapidly too.

Exactly how does this cultural change process unfold? And under what conditions might it occur more rapidly? Any useful theoretical model addressing these questions must do at least three things. First, it must include at least one variable representing a potentially-changeable personal attribute (e.g., an attitude) of each of the individuals who comprise a cultural population. Second, it must specify some means through which individuals are exposed to

\footnotetext{
${ }^{1}$ Lewin quoted this maxim in several publications in the $1940 \mathrm{~s}$, with no personal claim on its origin and with slightly different wording. For instance: "We should be aware of the value of theory. A business man once stated that "there is nothing as practical as a good theory"" (Lewin, 1943, p. 118). The revised phrasing - substituting "so" for "as" - appears to have become more popular within academic cultures.
} 
potential sources of social influence (e.g., friends or acquaintances who express different attitudes). And, third, it must specify a mechanism through which those individual attributes might change as a consequence of social influence - for instance, some sort of decision-rule governing the likelihood that individuals' attitudes do, or do not, change after obtaining information about others' attitudes.

Additionally, because cultural change occurs as a consequence of many acts of social influence unfolding over time, a useful theoretical model must represent this dynamic, cumulative process. This kind of model cannot simply represent a single snapshot of time; it must combine many hundreds of snapshots of social influence, linked together in coherent causal sequences, in order to meaningfully depict — and predict - consequences for cultural change.

This is a nontrivial conceptual challenge. The usual tools that psychologists use to express theoretical models (e.g., verbal statements and 2-dimensional depictions of causes and effects) are poorly suited to the task of predicting cultural consequences that emerge from the complex influence dynamics that unfold when whole populations of people interact with each other, repeatedly. More sophisticated theory-building methods are necessary (Smaldino, 2017, 2020; Smith \& Conrey, 2007). Computational models are particularly useful; and one particular kind of computational method-agent-based modeling - is especially useful.

\section{Agent-based Modeling of Social Influence Dynamics}

When applied to social psychological phenomena, agent-based modeling has been summarized like this: "Agent-based models (ABMs) are computational simulations in which artificial entities interact over time within customized environments. These entities (agents) are programmed to represent humans who behave in precisely specified ways" (Jackson et al., 2017, p. 387). In many such models, agents (i.e., people) can be assigned values that represent psychological attributes (e.g., personality traits, attitudes, etc.). They can also be programmed to interact with other agents and to simulate specific cognitive or behavioral outcomes resulting from those interactions. Thus, repeated iterations of a model represent repeated opportunities for individuals to interact with each other over time, while also simulating the cumulative effects that these repeated interactions have on population-level outcomes (e.g., changes in the frequencies and/or spatial distributions of specific attributes within the entire population).

Agent-based models have been used to simulate many different kinds of cultural phenomena (e.g., Nowak et al., 2016; Pfau, Kirley, \& Kashmia, 2013). A subset of these models have focused on social influence processes. One illustrative line of research employed agentbased models to articulate a theory of dynamic social impact, and its implications for the creation of culture (Harton \& Bourgeois, 2003; Latané, 1996). In these models, populations of agents (representing individual people) were arrayed in a grid-like pattern across a simulated social landscape. Each agent was randomly assigned several numerical values representing different potentially-changeable attributes (e.g., political attitudes, food preferences, etc.). These models then computationally simulated repeated opportunities for interpersonal interaction and social influence, and did so in a way that operationalized a small set of empirically-documented social psychological phenomena (e.g., people are inclined to adopt the beliefs and behaviors exhibited by the majority of people they interact with; people generally interact more frequently with their immediate neighbors than with people who are further away; some people are more influential than others). These computational inputs represented the conceptual guts of dynamic social impact theory. The outputs - changes in distributions of attributes across the social landscaperepresented predictions generated by the theory. Of particular note are two outputs that Latané 
(1996) called "clustering" and "correlation." As agents interacted with - and influenced - each other, their attributes (which were initially randomly distributed across the social landscape) coalesced into distinct clusters occupying different parts of the landscape. Additionally, as these clusters emerged, individuals' (initially uncorrelated) attributes became increasingly correlated, such that, within clusters, individuals became increasingly similar to each other on multiple attributes and, across clusters, individuals differed from each other on multiple attributes. (The latter phenomenon was driven, in part, by the tendency for especially influential individuals to exert their influence broadly, such that their neighbors increasingly become more similar to them, and to each other, in multiple ways. For this reason, attributes that bear no logical relation to each other - such as political attitudes and food preferences - may become equally emblematic of specific clusters of people in specific geographical regions.) Once formed, these clusters persisted, simulating the emergence of distinct cultures within a broader population.

Although mere words (such as those used in the preceding paragraph) can summarize the inputs and outputs of dynamic social impact theory, the utility of the theory-its ability to explain and predict cultural phenomena - is inseparable from the agent-based modeling methods that precisely specify the spatial and interpersonal dynamics of social influence, and reveal the consequences for emergent cultural-level phenomena. Indeed, given the bidirectional, nonlinear complexity of these social influence dynamics, rigorous computational methods are perhaps the only means through which the cultural consequences can actually be predicted.

\section{Applying this Framework to Research on Cultural Change}

Computational models of dynamic social impact theory reveal how social influence processes can lead to the emergence of rudimentary cultures. The same conceptual principles (emphasizing constraints on social interactions and the psychology of social influence), coupled to the same methodology (agent-based models) can be used to examine how existing cultures might continue to change over time. To do so, one must also simulate opportunities for cultural change-for example, the introduction of novel beliefs that have the potential to spread within a cultural population, and to possibly displace whatever beliefs were previously normative. While doing so, it is useful to be mindful of several facts about real-life cultures that have potentially important implications for the dynamics of social influence within those cultures.

One fact is that, just as individuals differ in their capacity to exert influence on others, they also differ in their susceptibility to be influenced by others. Therefore, when modeling the implications that social influence dynamics might have on cultural change, it is useful to simulate within-culture individual differences in influenceability. There is no single right way of doing so, because there are multiple mechanisms through which social influence occurs, and individuals may not be equally susceptible to all forms of social influence. One solution to this challenge is to model the distributional properties of broad dispositional traits that might plausibly affect individuals' susceptibility to various forms of influence.

Another fact that merits close consideration is the structure of social networks. Social networks - the complicated webs of dyadic connections among family members, friends, and acquaintances - represent the conduits through which interpersonal influence occurs, and potentially spreads (Kashima, Wilson, Lusher, Pearson, \& Pearson, 2013). When measured at the level of whole populations, social networks have a complex geometric structure that results, in part, from differences in the numbers of friends and acquaintances that different people have (Henrich \& Broesch, 2011). This complex structure can be summarized by a set of statistical parameters that reflect the density of interpersonal interconnections within a culture. One 
limitation of many models linking the psychology of social influence to cultural outcomes is that they do not simulate the complex social network structures of real-life cultural populations. If models can simulate those network structures more realistically, it is likely to pay off in the form of more realistic representations of the cultural consequences of social influence.

A third important fact about cultures is that they differ. Just as individuals differ in their susceptibility to social influence, cultures differ in overall levels of susceptibility to social influence. For instance, in more highly collectivistic cultures, there are higher mean levels of conformity to majority opinion (Bond \& Smith, 1996). Cultures also differ in social network structure. In more highly collectivistic cultures, individuals have smaller personal networks of friends and acquaintances (Liu, Chan, Qiu, Tov, \& Tong, 2018)—with the implication that population-level social network structures tend to be less densely-connected within those cultures. $^{2}$ These differences may affect the spread of social influence within cultural populations and, consequently, the dynamics of cultural change. Models that ignore cultural differences might mimic the dynamics of cultural change within some cultures, but not others. Indeed, it may be informative to deliberately model cultural differences (e.g., to construct multiple models designed to systematically simulate cultural differences in social network structure and susceptibility to social influence). By doing so, one may be able to gain new insights into the social influence dynamics that contribute to cultural changes, while also explaining and predicting cultural differences in the trajectory of those cultural changes.

\section{Illustrative Application: Agent-Based Models of Cultural Differences in Cultural Change}

Are these objectives actually obtainable? We believe so. In our own recent research, we used agent-based modeling methods to simulate the variables and processes summarized above, in order to investigate cultural differences in the pace of cultural change (Muthukrishna \& Schaller, 2020). Some elements of our models were adapted from previous research on the population-level consequences of interpersonal influence (e.g., models of dynamic social impact theory; models of cultural evolution). Other elements (including methods for modeling cultural differences in social network structure and susceptibility to social influence) are more novel, and were developed specifically to serve the objectives of this project. The outputs produced by these models - which represent rigorously-derived hypotheses about cultural differences in the pace of cultural change - may be of interest to scholars seeking to explain or predict cultural changes in real-life cultural populations. The modeling methods themselves may be of interest too. These methods are modular and flexible, and can potentially be used in future research to address a wide range of additional questions about cultural change. Therefore, for illustrative purposes, the sections below describe these models - and their outputs - in greater (but non-technical) detail.

\section{Modeling Cultures, Individual Differences, and Cultural Differences}

In order to model cultural change, one must create a model that simulates a cultural population. And in order to model cultural differences in cultural change, one must create multiple models that simulate multiple cultural populations that differ in one or more meaningful ways. The first phase of our agent-based modeling methodology was designed to do this.

\footnotetext{
2 There is an important conceptual distinction between (a) the network of connections within individuals' personal social networks and (b) the network of connections between all individuals within a cultural population. It is precisely because individuals' personal networks are smaller within collectivistic cultures that population-level network structures are less densely-connected within these cultures.
} 
Each model simulated a population of 900 individuals, each of whom was initially located on a space within a grid-like social landscape, and was assigned two numerical values representing two dispositional traits. One of these traits can be thought of as a behavioral manifestation of extraversion: the probability that the individual would make a new acquaintance when given the opportunity. This trait has implications for modeling social network structure, which occurred during the second phase of our models (described below). The other trait represented susceptibility to social influence. This trait has implications for modeling social influence outcomes, which occurred during the third phase of our models (also described below).

We assigned these traits to individuals in such a way as to operationalize both individual differences and cultural differences. Within each simulated culture, the distribution of trait values mimicked distributional properties of personality traits within real-life cultural populations (e.g., within-culture standard deviations of individual differences in extraversion; McCrae et al., 2005). Additionally, across the different cultures simulated by different models, the mean values of these traits differed, and did so in a way that mimicked the magnitude of actual cultural differences (e.g., between-culture mean differences in extraversion; McCrae et al., 2005). Details on the exact computational procedures can be found in Muthukrishna and Schaller (2020). The key point is this: By using these procedures, we simulated real-world individual differences in dispositions that have implications for social network structures and social influence outcomes, and also simulated real-world cultural differences in those dispositions.

\section{Modeling Social Network Structure}

The first phase of our models created cultural populations, but the individuals within those populations were still just isolated entities. Therefore, we implemented a second phase that was designed to provide each individual with a network of friends, and to do so in a way that simulated social network structures of real human societies. There are many computational algorithms that can produce network structure within agent-based models, but most such algorithms have no meaningful connection to human behavior (Schnettler, 2009). We developed a new method that simulated one way in which actual behavioral decision-making can, over time, lead to the emergence of realistic social network structure. This method also provided a means of simulating cultural differences in social network structure.

To do so, we programmed the model to operationalize two empirically-documented constraints on friendship formation. One of these constraints is spatial proximity: People more readily form connections with others who are closer in geographic space (Latané, Liu, Nowak, Bonevento, \& Zheng, 1995). The second constraint is personal disposition: Some individuals (e.g., extraverts) are more likely to form social connections than others, and thus have larger personal networks and friends and acquaintances (e.g., Selfhout et al., 2010). To simulate the effects of these variables in the formation of individuals' social networks, we programmed this phase of the model so that, across repeated iterations, individual agents had repeated opportunities to "move" across the social landscape, to "meet" other agents within that landscape, and to form friendships with them. During each iteration, individuals' movements (and meetings) were limited to adjacent spaces on the landscape, thus ensuring that the formation of friendships was constrained by spatial proximity. Additionally, these movements (and meetings) were probabilistic, and varied across individuals. During each iteration, the likelihood that an individual actually would move to an adjacent space (and meet another individual there) was a direct function of the extraversion value that had been assigned during the previous phase 
7

of the model. Thus, across multiple iterations of the model, individuals with relatively higher extraversion values acquired larger personal networks of friends.

These procedures had two important population-level consequences within the simulated cultures. First, as individual agents had repeated opportunities to form friendships with each other, realistic social network structures emerged within the simulated populations (as measured by a set of statistical indices commonly employed to describe network structure; for details, see Muthukrishna \& Schaller, 2020). And second, the density of these population-level network structures varied as a consequence of the mean level of extraversion within the population: In populations with higher mean levels of extraversion, the social network structure was denser (e.g., on average, individuals in these cultures were socially connected to a greater number of other individuals). Thus, these procedures allowed us to simulate cultural differences in the social network structures through which people exert social influence on each other.

Once realistic social network structures (and cultural differences in network structures) emerged in the simulated cultures, the second phase of the model was stopped and the emergent social network structures were retained for the final phase of our models - during which we simulated interpersonal influence dynamics and consequences for cultural change.

\section{Modeling Social Influence and Cultural Change}

The procedures employed in those first two phases set the stage for the third phase, during which the outcomes of primary conceptual interest occurred. It was during this phase that we (a) assigned potentially-changeable attributes (e.g. beliefs) to individual agents, (b) provided them with repeated opportunities for social interaction and social influence within their networks of friends, (c) simulated the psychological effects of these influence opportunities (e.g., changes in individuals' beliefs), and (d) assessed the emergent cultural-level implications (e.g. changes in the popularity of beliefs). Thus, we were able to systematically identify plausible ways in which cultural differences (in social network structure and in individuals' susceptibility to social influence) may affect the pace of cultural change.

In doing so, we ran tens of thousands of simulations. Two different subsets of simulations focused on two distinct kinds of cultural change: (a) consolidation of public opinion and (b) diffusion of initially unpopular beliefs.

Consolidation refers to the tendency for existing majorities to become bigger majorities over time (Latané, 1996) — exemplified by "bandwagon" effects in electoral politics —and provides a point of departure to illustrate the methods we employed during this phase of our models. To model the consolidation phenomenon - and to identify specific cultural differences that might affect the speed that it happens — we began by assigning an additional, potentiallychangeable attribute to each individual agent, with a value of either 0 or 1 . For the sake of illustration, one can think of these values as representing rival attitudes or behavioral tendencies (e.g., attitudes toward immigration policy, for instance, or inclinations to wear a facemask-or not - during a global pandemic). We assigned values in such a way as to ensure that, initially, the two values were approximately equally prevalent within the population. Subsequently, across repeated iterations of the model, individual agents had repeated opportunities to "interact" with other individuals within their own personal social networks (i.e., the friends that they had acquired during the previous phase of the model), to acquire information about the distribution of beliefs held by their friends, and to potentially change their own beliefs. We drew upon empirical evidence in the psychological literature to mathematically model multiple routes through which individuals might change their beliefs (allowing the possibility that individuals might conform to 
majority opinion as well as the possibility, at a substantially lower likelihood, that individuals might occasionally adopt a belief held by only a minority of their friends). Additionally, the likelihood of belief change was also a function of individual's own dispositional susceptibility to social influence. (For details, see Muthukrishna \& Schaller, 2020.)

Individual agents did sometimes change their beliefs and-as demonstrated across thousands of simulations - there were cumulative cultural-level implications. Although the two beliefs were initially equally popular, one belief inevitably became just a bit more enduringly popular; and once it did, it consequently became even more popular. Did this consolidation occur more quickly in some cultures, compared to others? Yes. In cultures with higher mean levels of influenceability, small majorities more rapidly consolidated into monolithic super-majorities.

Although consolidation represents a kind of cultural change, it is not a particularly momentous change. Diffusion of innovation refers to a more profound form of cultural change: An initially unpopular attribute (e.g., a belief held by a tiny minority of people) may spread within a population and perhaps - if it spreads widely enough — even becomes a new cultural norm (Rogers, 2003). Conformist tendencies inhibit the diffusion of unpopular beliefs; but, as research on minority influence reveals, initially unpopular beliefs can sometimes catch on, especially when initial adherents are themselves resistant to conformity pressure and have the opportunity to persistently express their unpopular beliefs to others (Moscovici, 1980).

To model this diffusion phenomenon - and to identify cultural differences that might affect whether initially unpopular beliefs do successfully spread-we conducted tens of thousands of simulations that used the methods described above to model the outcomes of repeated opportunities for social influence. There was one main difference: Rather than initially assigning the two different beliefs to equal numbers of individuals, we instead assigned one belief to the vast majority of individuals, while the other belief was initially highly unpopular. (Across different subsets of simulations, the unpopular belief was held by either just a single individual — a "lone ideologue" — or by that individual along with a small number of that individual's friends.) Additionally, in order to give that unpopular belief at least some chance to spread, we ensured that exactly one initial adherent of this unpopular belief had a large personal network of friends and was highly insusceptible to others' influence (for details, see Muthukrishna \& Schaller, 2020). After this initial assignment of beliefs, we simulated social interaction and social influence exactly as we did when modeling consolidation processes (described above), and examined the cultural consequences.

The outputs of these models showed that the initially unpopular belief often failed to spread, but sometimes it did. (Consistent with research on minority influence, its chances of spreading increased when an initial adherent was less susceptible to others' influence). Indeed, sometimes it spread so successfully that it was eventually adopted by the majority of other individuals, thus becoming the new cultural norm. Did this cultural transformation occur more readily in some simulated cultures, compared to others? Yes. It was especially likely to occur in cultures characterized by (a) less densely connected social network structures (i.e., lower mean levels of extraversion), and (b) higher mean levels of susceptibility to social influence.

The latter effect may seem counterintuitive, especially in light of the consolidation results described above. How can populations that exhibit more rapid consolidation of an existing norm also be more prone to the abandonment of that norm in favor of a new one? The answer lies in the tendency for individuals who are more susceptible to social influence to be more susceptible to any form of social influence. When people are less committed to maintaining their own preexisting beliefs and are more willing to entertain the beliefs expressed by others, they are not 
only more susceptible to conformity pressure, they are also more susceptible to persuasion by an idiosyncratic friend who persistently expresses an unpopular belief. This is especially likely to occur under conditions in which they have a relatively small personal social network (and thus relatively small number of other friends who express more conventional beliefs). It is for this reason that, according to our models, cultural transformation occurs more readily within cultures in which people are, on average, less extraverted and more susceptible to social influence. Both characteristics are associated with more highly collectivistic cultures.

Because these effects are computational outputs, it is important to ask whether the effects are peculiar to an idiosyncratic set of computational inputs. Our results indicated that they are not. Across the tens of thousands of simulations, we systematically varied many of the parameters in our models in order to mimic a range of plausible psychological assumptions. Reassuringly, we found that the effects summarized above were robust across these variations. ${ }^{3}$ It is also reassuring that conceptually similar results emerged independently from a separate modeling project. De et al. (2018) employed rather different methods to model cultural differences in individuals' conformist tendencies, and found that (a) high-conformity cultures showed more initial resistance to norm change and (b) when norms eventually did change, they changed more rapidly. Thus, multiple methodologically-distinct but conceptuallycomplementary models imply that, even though collectivistic cultures may show speedier consolidation of existing cultural norms, these same cultures may also be more likely to experience the kind of transformative change that occurs when populations rapidly abandon old norms in favor of new ones.

\section{Evidence, Explanation and Prediction}

Because the latter hypothesis is nonobvious and perhaps even counterintuitive, it would be sensible to view it skeptically in the absence of empirical evidence. In fact, several pieces of evidence do provide some empirical support for it. One set of studies compared a prototypically individualistic country (USA) with a prototypically collectivistic country (Japan), and found that popularities of books, music, and baby names changed more rapidly in the latter cultural context (Wilken, Miyamoto, \& Uchida, 2011). Another study examined the rates at which innovative products (e.g., hand calculators, color televisions, microwave ovens) penetrated consumer markets within different European countries during the 1970s and 1980s. Results revealed that, after controlling for economic differences, these innovations were popularly adopted more quickly in more highly collectivistic countries (Dwyer, Mesak, \& Hsu, 2005). Books and baby names and microwave ovens are very different kinds of cultural things and so, in the absence of an explanatory theory, these findings might seem to be little more than isolated examples of curious cross-national differences. Our models, along with those of De et al. (2018), offer a theoretical framework that can explain and integrate these findings, suggesting that - because of underlying cultural differences in the population-level dynamics of social influence - they reflect a general tendency for more highly collectivistic cultures to be more susceptible to rapid change.

This framework may not only help to explain past patterns of cultural change, it might also be useful for forecasting future patterns of change. Our models did not simulate specific

\footnotetext{
${ }^{3}$ For example: Because people preferentially form social connections with attitudinally similar others, and because this preference varies across cultures (e.g., Heine, Foster, \& Spina, 2009), our models systematically varied whether or not individuals' initial beliefs were independent of the beliefs of their friends. The results summarized above emerged regardless. See Muthukrishna \& Schaller (2020) for additional details regarding additional tests of robustness.
} 
cultures or specific time-frames, and so cannot predict exact locations or speeds of future cultural changes; but, by referring to empirical research describing cross-national differences in traits and values and susceptibilities to social influence (e.g., Gelfand et al., 2011; Hofstede, 2001; McCrae et al., 2005), these models do predict that — all else being equal—-truly transformative cultural change will be relatively more likely to occur within countries that have population profiles of low extraversion and high influenceability. For example: Brazil and Peru are similar along many dimensions, but some research (Hofstede, 2001; McCrae et al., 2005) indicates that Peruvians are relatively less extraverted and more collectivistic (and, by implication, more susceptible to social influence). If those differences are real and persistent, our models predict that the coming decades will witness more truly transformative cultural changes in Peru than Brazil.

\section{Additional Applications to Additional Questions about Cultural Change}

Muthukrishna and Schaller (2020) focused on just two specific cultural differences that might plausibly affect the temporal trajectories of cultural change. The same conceptual framework and modeling methods could be applied to study the effects of other cultural differences that might also have implications for interpersonal influence and cultural change. For instance, it may be informative to model implications of specific kinds of cultural values - such as the broad value dimensions identified by Schwartz (2011), or more highly-focused values of the sort measured on the World Values Survey (which, when combined, reveal fundamental similarities and differences between cultures; Muthukrishna, Bell, et al. 2020). Cultural differences in motives and goals might also matter. Individuals' susceptibility to social influence can be affected by epistemic needs (e.g., need for cognitive closure) that vary not only across individuals but across cultures too (Fu, Morris, Lee, Chao, Chiu, \& Hong, 2007). Additionally, the scope of individuals' social interactions - and thus their opportunities to exert influence on and be influenced by others - may vary depending on affiliative motives that incline individuals toward interpersonal approach behavior (Maner, DeWall, Baumeister, \& Schaller, 2007), or on self-protective and disease-avoidant motives that make people more socially wary (Neuberg, Kenrick, \& Schaller, 2011). The latter motives have implications for conformity behavior too (Murray \& Schaller, 2012). Recent research documents cultural similarities and differences in mean levels of these motives (Ko et al., 2020) — results that can potentially inform future computational models of cultural differences in cultural change.

This framework might also serve as a basis for future models that focus in a more nuanced way on different kinds of attitudes and beliefs, and - more generally — different kinds of cultural norms. In the computational models described above, individual agents did not actually have specific attitudes or beliefs about specific things; rather, these agents were attached to numerical values that could represent any attitude or belief (or, really, any attribute of any kind that might be subject to social influence). In real life, some attitudes and beliefs are more amenable than others to social influence (e.g., Bourgeois, 2002) and it would be sensible to take these differences into account when predicting whether any particular attitude or belief is likely to become, or remain, culturally popular. More generally, models of cultural change are likely to produce more useful predictions if they realistically operationalize the extent that particular cultural norms are, or are not, responsive to the psychology of social influence.

Similarly, because communication is the medium through which interpersonal influence operates, it could be useful to carefully consider the extent to which different attitudes and beliefs and other kinds of information are likely to be communicated from person to person. 
Research on contemporary cultural myths - urban legends - shows that the more disgusting an urban legend is, the more motivated people are to talk about it, and the more likely it is to become widely known (Heath, Bell, \& Strenberg, 2001). The specific contents of widespread ethnic stereotypes appear also be influenced by interpersonal communication processes: People are more inclined to talk about some stereotypic traits than others, and these differences predict which traits do or do not persist in popular stereotypes (Schaller, Conway, \& Tanchuk, 2001). To the extent that one can reliably estimate the "communicability" of attitudes or beliefs or other pieces of knowledge, and incorporate those estimates into models of dynamic social influence, one may be able to generate better predictions about the extent to which these things are likely to become more, or less, culturally normative over time.

Additionally, different kinds of information may be communicated with different levels of effectiveness via different channels of communication (e.g., face-to-face conversation, different forms of electronic communication); and different constraints and decision-rules may govern the formation of social connections within different kinds of interactive environments (e.g., neighborhoods, workplaces, online social media). It would be useful to incorporate these variables too into models of social influence and cultural change.

Because this framework can be adapted to model any kind of cultural change, it can potentially be applied to specific cultural changes of substantial real-world consequenceincluding changes in governance (e.g., the rise of authoritarianism within democratic societies), changes in norms governing the provision of public goods (e.g., norms pertaining to bribery and corruption; Muthukrishna et al., 2017), changes in harmful cultural traditions (e.g., female genital mutilation; Efferson, Vogt, \& Fehr, 2020), and changes in public opinion regarding public health policies (e.g., adoption of, or resistance to, wearing of facemasks during a pandemic; Muthukrishna, 2020). This framework might also be helpful in understanding specific changes that are so psychologically and culturally fundamental as to affect the process of cultural change itself. Consider, for example, the recent worldwide historical trend toward increased individualism and decreased collectivism (Hamamura, 2012; Santos et al., 2017). This trend may be partially attributable to historical changes in ecological circumstances (Santos et al., 2017), but it might also be partially attributable to social influence dynamics. Because collectivistic values are associated with greater susceptibility to social influence, collectivists may be more readily influenced by others' individualistic values, whereas individualists are less likely to adopt others' collectivistic values. As a consequence, over time, individualistic values may spread within an initially collectivistic culture, whereas collectivistic values are less likely to catch on within an individualistic culture. If so, then the pace of change in individualistic and collectivistic values may be moderated by other population-level variables that affect social influence dynamics (e.g., social network structure; Muthukrishna \& Schaller, 2020). This is just crude conjecture, of course, which cannot be trusted to deliver meaningful hypotheses about the outcomes of complex dynamical systems (especially those that have feedback loops of the kind implied here). To do that, rigorous modeling must be done.

Additional insights may accrue from the integration of ecological variables into this conceptual framework. Just as there are multiple mechanisms through which ecological variation may produce cultural variation (Schaller \& Murray, 2011, 2012), there are multiple mechanisms through which ecological changes may produce cultural changes, and some of those mechanisms are entirely independent of social influence processes. But there is reason to believe that some forms of ecological variation may affect population-wide susceptibilities to social influence, or the social network structures through which social influence occurs (e.g., Oishi \& Kesebir, 
2012), or both. Research on pathogen prevalence provides an illustrative example. Pathogen prevalence is correlated with cultural variation in both conformity and extraversion (Murray, Trudeau, \& Schaller, 2011; Schaller \& Murray, 2008)_findings that are complemented by experiment evidence linking increased disease threat to increased conformity and to decreased extraversion (Mortensen et al., 2010; Murray \& Schaller, 2012). The implication is that changes in the ecological profiles of infectious diseases - such as when historically endemic diseases are eliminated or when novel epidemics arise - may affect the social influence dynamics modeled by Muthukrishna and Schaller (2020), and thus affect the pace of cultural change.

Sng et al. (2019) identify additional ecological variables that might productively be incorporated into models of dynamical social influence and cultural change. One such variable is sex ratio. There are sex differences in susceptibilities to specific forms of social influence (e.g., Cross, Brown, Morgan, \& Laland, 2017), and men and women also differ in friendship patterns (Dunbar, 2018); consequently, the relative ratio of men to women within a population may affect the population-level consequences of interpersonal influence. Another variable that might matter is the mean number of offspring produced per person. This variable substantially affects the demographic profile of a cultural population and, since adults are likely to exert greater influence on children than the reverse, may also affect the cumulative consequences that accrue from repeated opportunities for interpersonal influence. Mobility might also matter. Cultures vary in mean levels of both residential and relational mobility, which-independent of cultural differences in personality traits - have implications for interpersonal interactions, friendship strategies and social network structures (Choi \& Oishi, 2020; Thomson et al., 2018). Consequently, these cultural differences too may affect trajectories of cultural change. Again, these thoughts are merely conjectures; they await rigorous conceptual development and systematic modeling.

\section{Final Thoughts}

Just as there is more than one kind of process through which cultures change, so too there is more than one way of modeling cultural change. Different kinds of models serve different functions. For example, statistical models - such as time-series models — can be used to describe specific cultural changes that occurred during specific periods of time during the historical past. Consequently, they provide a data-driven tool for predicting analogous changes that might occur under analogous circumstances in the future. The utility of these models depends on the existence of reliable historical data; and, while time-series models can sometimes identify specific variables that statistically predict specific cultural changes (e.g., Varnum \& Grossmann, 2017b), they do not explain, in psychological terms, how those changes might have occurred. To serve that function, some sort of theoretical model is required.

Some theoretical models serve that function better than others. Just as empirical observations have greater value when generated by methods that meet high standards of scientific rigor, theoretical models offer more useful explanations and predictions when articulated in ways that are logically rigorous, systematic, objective and transparent (Grahek, Schaller, \& Tackett, in press; Muthukrishna \& Henrich, 2019; Schaller, 2016; Smaldino, 2017). Many psychological theories fall short of these standards because of unstated assumptions and implicit leaps in logic, or because they are expressed with words that have inherent ambiguity in meaning. Formal modeling methods offer a way to overcome these shortcomings (Muthukrishna \& Henrich, 2019; Smaldino, 2017). Some formal models are simply systems of mathematical 
equations. Others, exemplified by the agent-based models that we have described here, harness the power of computers to simulate the complex dynamic interplay between the characteristics of individuals and the characteristics of the populations that those individuals comprise. These modeling methods offer indispensable tools for the psychological study of cultural change.

And these tools are not so hard to use! People are sometimes scared off by the jargon and mathematical details that appear in many modeling publications; but this superficial abstruseness belies the fact that, with only a modest investment of time and effort, anyone can construct a computational model. Lots of helpful resources are readily available to novice modelers. Smaldino (2017) provides a plainspoken introduction to the principles and methods of computational modeling; and Smaldino (2020) provides lucid beginner-level guidance on how to actually construct such models. For agent-based modeling of social psychological processes in particular, Smith \& Conrey (2007) and Jackson et al. (2017) offer excellent introductions and plenty of practical guidance. For instance, Jackson et al. (2017) provides a step-by-step recipe for building an agent-based model, accompanied by illustrative examples. Additional examples can be found in recent book on Computational Social Psychology (Vallacher, Read, \& Nowak, 2017). Also useful are several books (Kokko, 2007; McElreath \& Boyd, 2008; Otto \& Day, 2011) that, although directed primarily toward students in other disciplines, provide instruction that is readily applicable to models of cultural change. Other resources can be found online. For instance, the Cultural Evolution Society hosts self-paced tutorials (http://www.dysoc.org/cesmodules/) that can help anyone to develop the skillset necessary to construct models of cultural dynamics and cultural change.

That skillset sometimes includes some proficiency at computer programming, but advanced programming skills are often not required. Many influential models of cultural dynamics require no programming acumen beyond what a typical teenager might learn in a highschool computer class (e.g., Kenrick, Li, \& Butner, 2003; Nowak, Szamrej, \& Latané, 1990), and free software packages serve the needs of novices with no formal programming experience at all. One such platform is NetLogo (https://ccl.northwestern.edu/netlogo/), which provides tutorials and a library of existing models that, through thoughtful modification, can serve as ideal building blocks for new and different models that address new and different research questions.

Some modeling projects pose larger technical challenges than others, of course. Our own work (Muthukrishna \& Schaller, 2020, described above) included novel elements-such as the modeling of social network structure - that required bespoke programming beyond the skillset of most beginners. But such models can serve as a starting point for modification without understanding all aspects of the algorithm. And if this too is beyond one's skillset, it still need not be a barrier. Any challenge posed by lack of expertise can be solved by a single all-purpose trick: Collaboration. Again, our own work is illustrative. Regardless of whatever other merits it might or might not have, Muthukrishna and Schaller (2020) offers irrefutable evidence that even someone who isn't adept at computer programming (at all!) can complete a publishable computational modeling project, if they collaborate with someone who is. ${ }^{4}$

\footnotetext{
${ }^{4}$ Schaller wants to make it absolutely clear: Muthukrishna is the one who is.
} 


\begin{abstract}
Author Note
This work was supported by research funding from the Social Sciences and Humanities Research Council of Canada (Insight Development Grant \#430-2018-00218).
\end{abstract}

\title{
References
}

Berger, J. (2013). Contagious: Why things catch on. New York: Simon \& Schuster.

Bond, R., \& Smith, P. B. (1996). Culture and conformity: A meta-analysis of studies using Asch's (1952b, 1956) line judgment task. Psychological Bulletin, 119, 111-137. https://doi.org/10.1037/00332909.119.1.111

Bourgeois, M. J. (2002). Heritability of attitudes constrains dynamic social impact. Personality and Social Psychology Bulletin, 28, 1063-1072. https://doi.org/10.1177/01461672022811005

Choi, H, \& Oishi, S. (2020). The psychology of residential mobility: A decade of progress. Current Opinion in Psychology, 32, 72-75. https://doi.org/10.1016/j.copsyc.2019.07.008

Cross, C. P., Brown, G. R., Morgan, T. J. H., \& Laland, K. N. (2017). Sex differences in confidence influence patterns of conformity. British Journal of Psychology, 108, 655-667. https://doi.org/10.1111/bjop.12232

De, S., Nau, D. S., Pan, X., \& Gelfand, M. J. (2018). Tipping points for norm change in human cultures. In Thomson R., Dancy C., Hyder A., Bisgin H. (Eds), Social, Cultural, and Behavioral Modeling. SBP-BRiMS 2018. Lecture Notes in Computer Science, 10899, 61-69. Springer. https://doi.org/10.1007/978-3-319-93372-6 7

Dunbar, R. I. M. (2018). The anatomy of friendship. Trends in Cognitive Sciences, 22, 32-51. https://doi.org/10.1016/j.tics.2017.10.004

Dwyer, S., Mesak, H., \& Hsu, M. (2005). An exploratory examination of the influence of national culture on cross-national product diffusion. Journal of International Marketing, 13(2), 1-27. https://doi.org/10.1509/jimk.13.2.1.64859

Efferson, C., Vogt, S., \& Fehr, E. (2020). The promise and the peril of using social influence to reverse harmful traditions. Nature Human Behaviour, 4, 55-68. https://doi.org/10.1038/s41562-019-0768-2

Eriksson, K., Enquist, M., \& Ghirlanda, S. (2007). Critical points in current theory of conformist social learning. Journal of Evolutionary Psychology, 5, 67-87. https://doi.org/10.1556/jep.2007.1009

Fu, J. H.-y., Morris, M. W., Lee, S.-1., Chao, M., Chiu, C.-y., \& Hong, Y.-y. (2007). Epistemic motives and cultural conformity: Need for closure, culture, and context as determinants of conflict judgments. Journal of Personality and Social Psychology, 92, 191-207. https://doi.org/10.1037/00223514.92.2.191

Gelfand, M. J., et al., (2011). Differences between tight and loose cultures: A 33-nation study. Science, 332, 1100-1104. https://doi.org/10.1126/science.1197754

Greenwald, A. G. (2012). There is nothing so theoretical as a good method. Perspectives on Psychological Science, 7, 99-108. https://doi.org/10.1177/1745691611434210

Grossmann, I., \& Varnum, M. E. (2015). Social structure, infectious diseases, disasters, secularism, and cultural change in America. Psychological Science, 26, 311-324. https://doi.org/10.1177/0956797614563765

Grahek, I., Schaller, M., \& Tackett, J. L. (in press). Anatomy of a psychological theory: Integrating construct validation and computational modeling methods to advance theorizing. Perspectives on Psychological Science.

Hamamura, T. (2012). Are cultures becoming individualistic? A cross-temporal comparison of individualism-collectivism in the United States and Japan. Personality and Social Psychology Review, 16, 3-24. https://doi.org/10.1177/1088868311411587

Harton, H. C., \& Bourgeois, M. J. (2003). Cultural elements emerge from dynamic social impact. In M. Schaller \& C. S. Crandall (Eds.), The psychological foundations of culture (pp. 41-75). New York: Psychology Press. 
Heath, C., Bell, C., \& Sternberg, E. (2001). Emotional selection in memes: The case of urban legends. Journal of Personality and Social Psychology, 81, 1028-1041. https://doi.org/10.1037/00223514.81.6.1028

Heine, S. J., Foster, J. A. B., \& Spina, R. (2009). Do birds of a feather universally flock together? Cultural variation in the similarity-attraction effect. Asian Journal of Social Psychology, 12, 247-258. https://doi.org/10.1111/j.1467-839X.2009.01289.x

Henrich, J., \& Broesch, J. (2011). On the nature of cultural transmission networks: Evidence from Fijian villages for adaptive learning biases. Philosophical Transactions of the Royal Society B, 366, 11391148. https://doi.org/10.1098/rstb.2010.0323

Hofstede, G. (2001) Culture's consequences (2nd ed.) London: Sage.

Jackson, J. C., Rand, D., Lewis, K., Norton, M. I., \& Gray, K. (2017). Agent-based modeling: A guide for social psychologists. Social Psychological and Personality Science, 8, 387-395. https://doi.org/10.1177/1948550617691100

Kashima, Y., Wilson, S., Lusher, D., Pearson, L. J., \& Pearson, C. (2013). The acquisition of perceived descriptive norms as social category learning in social networks. Social Networks, 35, 711-719. https://doi.org/10.1016/j.socnet.2013.06.002

Kenrick, D. T., Li, N. P., \& Butner, J. (2003). Dynamical evolutionary psychology: Individual decision rules and emergent social norms. Psychological Review, 110, 3-28. https://doi.org/10.1037/0033295X.110.1.3

Ko, A., Pick, C. M., Kwon, J. Y., Barlev, M., Krems, J. A., Varnum, M. E. W., et al. (2020). Family matters: Rethinking the psychology of human social motivation. Perspectives on Psychological Science, 15, 173-201. https://doi.org/10.1177/1745691619872986

Jackson, J. C., Rand, D., Lewis, K., Norton, M. I., \& Gray, K. (2017). Agent-based modeling: A guide for social psychologists. Social Psychological and Personality Science, 8, 387-395. https://doi.org/10.1177/1948550617691100

Kokko, H. (2007). Modelling for field biologists and other interesting people. Cambridge University Press.

Latané, B. (1996). Dynamic social impact: The creation of culture by communication. Journal of Communication, 46(4), 13-25. https://doi.org/10.1111/j.1460-2466.1996.tb01501.x

Latané, B., Liu, J. H., Nowak, A., Bonevento, M., \& Zheng, L. (1995). Distance matters: Physical space and social impact. Personality and Social Psychology Bulletin, 21, 795-805. https://doi.org/10.1177/0146167295218002

Lewin, K. (1943). Psychology and the process of group living. The Journal of Social Psychology, 17, 113-131. https://doi.org/10.1080/00224545.1943.9712269

Liu, P., Chan, D., Qiu, L., Tov, W., \& Tong, V. J. C. (2018). Effects of cultural tightness-looseness and social network density on expression of positive and negative emotions: A large-scale study of impression management by Facebook users. Personality and Social Psychology Bulletin, 44, 15671581. https://doi.org/10.1177/0146167218770999

Maner, J. K., DeWall, C. N., Baumeister, R. F., \& Schaller, M. (2007). Does social exclusion motivate interpersonal reconnection? Resolving the "porcupine problem." Journal of Personality and Social Psychology, 92, 42-55. https://doi.org/10.1037/0022-3514.92.1.42

McCrae, R. R., Terracciano, A., \& 79 Members of the Personality Profiles of Cultures Project. (2005). Personality profiles of cultures: Aggregate personality traits. Journal of Personality and Social Psychology, 89, 407-425. https://doi.org/10.1037/0022-3514.89.3.407

McElreath, R., \& Boyd, R. (2008). Mathematical models of social evolution: A guide for the perplexed. University of Chicago Press.

Mortensen, C. R., Vaughn Becker, D., Ackerman, J. M., Neuberg, S. L., \& Kenrick, D. T. (2010). Infection breeds reticence: The effects of disease salience on self-perceptions of personality and behavioral avoidance tendencies. Psychological Science, 21, 440447. https://doi.org/10.1177/0956797610361706 
Moscovici, S. (1980). Toward a theory of conversion behavior. Advances in Experimental Social Psychology, 13, 209-239. https://doi.org/10.1016/S0065-2601(08)60133-1

Murray, D. R., \& Schaller, M. (2012). Threat(s) and conformity deconstructed: Perceived threat of infectious disease and its implications for conformist attitudes and behavior. European Journal of Social Psychology, 42, 180-188. https://doi.org/10.1002/ejsp.863

Murray, D. R., Trudeau, R., \& Schaller, M. (2011). On the origins of cultural differences in conformity: Four tests of the pathogen prevalence hypothesis. Personality and Social Psychology Bulletin, 37, 318-329. https://doi.org/10.1177/0146167210394451

Muthukrishna, M. (2020). Long read: Cultural evolution, Covid-19, and preparing for what's next. LSE Business Review (22 April, 2020). http://eprints.lse.ac.uk/id/eprint/104402

Muthukrishna, M., Bell, A. V., Henrich, J., Curtin, C. M., Gedranovich, A., McInerney, J., \& Thue, B. (2020). Beyond western, educated, industrial, rich, and democratic (WEIRD) psychology: Measuring and mapping scales of cultural and psychological distance. Psychological Science, 31, 678-701. https://doi.org/10.1177/0956797620916782

Muthukrishna, M., Francois, P., Pourahmadi, S., \& Henrich, J. (2017). Corrupting cooperation and how anti-corruption strategies may backfire. Nature Human Behavior, 1, 0138. https://doi.org/10.1038/s41562-017-0138

Muthukrishna, M., \& Henrich, J. (2019). A problem in theory. Nature Human Behaviour, 3, 221-229. https://doi.org/10.1038/s41562-018-0522-1

Muthukrishna, M., Henrich, J. \& Slingerland, E. (2021). Psychology as a historical science. Annual Review of Psychology, 72, XXX-XXX. https://doi.org/10.1146/annurev-psych-082820-111436

Muthukrishna, M., \& Schaller, M. (2020). Are collectivistic cultures more prone to rapid transformation? Computational models of cross-cultural differences, social network structure, dynamic social influence, and cultural change. Personality and Social Psychology Review, 24, 103-120. https://doi.org/10.1177/1088868319855783

Neel, R., Kenrick, D. T., White, A. E., \& Neuberg, S. L. (2016). Individual differences in fundamental social motives. Journal of Personality and Social Psychology, 110, 887907. https://doi.org/10.1037/pspp0000068

Neuberg, S. L., Kenrick, D. T., \& Schaller, M. (2011). Human threat management systems: Selfprotection and disease avoidance. Neuroscience and Biobehavioral Reviews, 35, 1042-1051. https://doi.org/10.1016/j.neubiorev.2010.08.011

Nowak, A., Gelfand, M. J., Borkowski, W., Cohen, D., \& Hernandez, I. (2016). The evolutionary basis of honor cultures. Psychological Science, 27, 12-24. https://doi.org/10.1177/0956797615602860

Nowak, A., Szamrej, J., \& Latané, B. (1990). From private attitude to public opinion: A dynamic theory of social impact. Psychological Review, 97, 362-376. https://doi.org/10.1037/0033-295X.97.3.362

Oishi, S., \& Kesebir, S. (2012). Optimal social-networking strategy is a function of socioeconomic conditions. Psychological Science, 23, 1542-1548. https://doi.org/10.1177/0956797612446708

Otto, S. P., \& Day, T. (2011). A biologist's guide to mathematical modeling in ecology and evolution. Princeton University Press.

Pfau, J., Kirley, M., \& Kashima, Y. (2013). The co-evolution of cultures, social network communities, and agent locations in an extension of Axelrod's model of cultural dissemination. Physica A: Statistical Mechanics and its Applications, 392, 381-391. https://doi.org/10.1016/j.physa.2012.09.004

Rogers, E. M. (2003). Diffusion of innovations (5th ed.). New York: Free Press.

Santos, H. C., Varnum, M. E. W., \& Grossmann, I. (2017). Global increases in individualism. Psychological Science, 28, 1228-1239. https://doi.org/10.1177/0956797617700622

Schaller, M. (2016). The empirical benefits of conceptual rigor: Systematic articulation of conceptual hypotheses can reduce the risk of non-replicable results (and facilitate novel discoveries too). Journal of Experimental Social Psychology, 66(5), 107-115. https://doi.org/10.1016/j.jesp.2015.09.006

Schaller, M., Conway, L. G., III, \& Tanchuk, T. L. (2002). Selective pressures on the once and future contents of ethnic stereotypes: Effects of the communicability of traits. Journal of Personality and Social Psychology, 82, 861-877. https://doi.org/10.1037/0022-3514.82.6.861 
Schaller, M., \& Murray, D. R. (2008). Pathogens, personality, and culture: Disease prevalence predicts worldwide variability in sociosexuality, extraversion, and openness to experience. Journal of Personality and Social Psychology, 95, 212-221. https://doi.org/10.1037/0022-3514.95.1.212

Schaller, M., \& Murray, D. R. (2011). Infectious disease and the creation of culture. In M. J. Gelfand, C.y. Chiu, \& Y.-y. Hong (Eds.), Advances in culture and psychology (Vol. 1, pp. 99-151). New York: Oxford University Press. https://doi.org/10.1093/acprof:oso/9780195380392.001.0001

Schaller, M., \& Murray, D. R. (2012). Mechanisms by which parasites influence cultures, and why they matter. Behavioral and Brain Sciences, 35, 91-92. https://doi.org/10.1017/S0140525X11001038

Schwartz, S. H. (2011). Values: Cultural and individual. In F. J. R. van de Vijver, A. Chasiotis, \& S. M. Breugelmans (Eds.), Fundamental questions in cross-cultural psychology (p. 463-493). Cambridge University Press. https://doi.org/10.1017/CBO9780511974090.019

Schnettler, S. (2009). A structured overview of 50 years of small-world research. Social Networks, 31, 165-178. https://doi.org/10.1016/j.socnet.2008.12.004

Selfhout, M., Burk, W., Branje, S., Denissen, J., Van Aken, M., \& Meeus, W. (2010). Emerging late adolescent friendship networks and Big Five personality traits: A social network approach. Journal of Personality, 78, 509-538. https://doi.org/10.1111/j.1467-6494.2010.00625.x

Smaldino, P. E. (2017). Models are stupid, and we need more of them. In R. Vallacher, S. Read, \& A. Nowak (Eds.), Computational Social Psychology (pp. 311-331). Routledge.

Smaldino, P. E. (2020). How to translate a verbal theory into a formal model. Social Psychology, 51, 207-218. https://doi.org/10.1027/1864-9335/a000425

Smith, E. R., \& Conrey, F. R. (2007). Agent-based modeling: A new approach for theory building in social psychology. Personality and Social Psychology Review, 11, 87-104. https://doi.org/10.1177/1088868306294789

Sng O., Neuberg, S. L., Varnum, M. E. W., \& Kenrick, D.T. (2018). The behavioral ecology of cultural psychological variation. Psychological Review, 125, 714-743. https://doi.org/10.1037/rev0000104

Thomson, R., Yuki, M., Talhelm, T., Schug, J., Kito, M., Ayanian, A. H., et al. (2018). Relational mobility predicts social behaviors in 39 countries and is tied to historical farming and threat. Proceedings of the National Academy of Sciences of the United States of America, 115, 7521-7526. http://dx.doi.org/10.1073/pnas.1713191115

Vallacher, R. R., Read, S. J., \& Nowak, A. (Eds.). (2017). Computational social psychology. New York: Routledge.

Varnum, M. E., \& Grossmann, I. (2017a). Cultural change: The how and the why. Perspectives on Psychological Science, 12, 956-972. https://doi.org/10.1177/1745691617699971

Varnum, M., \& Grossmann, I. (2017b). Pathogen prevalence is associated with cultural changes in gender equality. Nature Human Behavior, 1, 0003. https://doi.org/10.1038/s41562-016-0003

Wilken, B., Miyamoto, Y., \& Uchida, Y. (2011). Cultural influences on preference consistency: Consistency at the individual and collective levels. Journal of Consumer Psychology, 21, 346-353. https://doi.org/10.1016/j.jcps.2011.03.005 


\section{Table 1.}

This article employs terminology-referring to population-level phenomena and methods employed to study those phenomena-that may be relatively unfamiliar to many readers of this journal. This table identifies, and briefly explains, several such terms.

Agent-based model. A type of computational model that simulates the actions and interactions of multiple individual entities (agents), producing outputs that reflect the cumulative consequences of those actions and interactions. In the present context, agents represent individual people; and agent-based modeling serves as an analytically rigorous method for generating hypotheses identifying specific ways in which, over time, individuals' actions and interactions have population-level consequences (e.g., changes in cultural norms).

Consolidation. A population-level phenomenon in which an attribute associated with the majority of people becomes, over time, associated with an increasingly larger majority of people. Through this form of cultural change, an initially normative attribute becomes even more highly normative within a culture.

Diffusion of innovation. A population-level phenomenon in which an initially unpopular attribute spreads and, over time, becomes associated with an increasingly larger percentage of people. Through this form of cultural change, an initially counter-normative attribute may be transformed into a new cultural norm.

Social network. A network of relationships between individuals. Social network analyses may focus on individuals' social networks, or on population-level social network structure. An individual person's social network refers to the specific set of other individuals with whom that person has a social connection (e.g., friendship) and to the connections among those individuals. The social network structure of an entire population (e.g., a culture) refers to the complete set of individuals within that population and to the network of social connections among them all. 